\title{
A reporter system for assaying influenza virus RNP functionality based on secreted Gaussia luciferase activity
}

Wenfei Zhu', Jianfang Zhou', Kun Qin, Ning Du, Liqi Liu, Zaijiang Yu, Yun Zhu, Wenhong Tian, Xiaobing Wu, Yuelong Shu ${ }^{*}$

\begin{abstract}
Background: Influenza A virus can infect a wide variety of animal species including humans, pigs, birds and other species. Viral ribonucleoprotein (VRNP) was involved in genome replication, transcription and host adaptation. Currently, firefly luciferase (Fluc) reporter system was used in VRNP functional assay. However, its limitation for the testing by virus infection resulted in an increased need for rapid, sensitive, and biosafe techniques. Here, an influenza A virus UTR-driven gene reporter for VRNP assay based on secreted Gaussia luciferase (Gluc) activity was evaluated.

Results: By measuring Gluc levels in supernatants, reporter gene activity could be detected and quantitated after either reconstitution of influenza A virus polymerase complex or viral infection of 293T and A549 cells, respectively. As compared with Fluc reporter, Gluc-based reporter was heat-tolerant $\left(65^{\circ} \mathrm{C}\right.$ for $\left.30 \mathrm{~min}\right)$ and produced 50 -fold higher bioluminescent activity at $24 \mathrm{~h}$ posttransfection. Signals generated by Gluc reporter gene could be detected as early as $6 \mathrm{~h}$ post-infection and accumulated with time. Testing by viral infection, stronger signals were detected by Gluc reporter at a $\mathrm{MOI}$ of 0.001 than that of 1 and the effects of PB2-627K/E or amantadine on influenza VRNP activity were elucidated more effectively by the Gluc reporter system.
\end{abstract}

Conclusions: This approach provided a rapid, sensitive, and biosafe assay of influenza VRNP function, particularly for the highly pathogenic avian influenza viruses.

\section{Background}

Bioluminescence reporters such as firefly, click-beetle, Renilla, Gaussia, Cypridina luciferases, and calciumactivated photoproteins are widely used in molecular biology. Of them, the firefly luciferase (Fluc) from the North American firefly, first cloned and sequenced by Wet et al. in 1985, is the most commonly used [1]. Gaussia luciferase (Gluc), naturally secreted from the marine copepod, was reported by Tannous in 2005 and found to be $\sim 2000$-fold more sensitive than either the Fluc or Renilla reniformis luciferases [2]. Expression levels in growth medium are readily quantifiable by addition of coelenterazine and luminometry [2]. To

\footnotetext{
* Correspondence: yshu@cnic.org.cn

† Contributed equally

State Key Laboratory for Molecular Virology and Genetic Engineering,

Chinese National Influenza Center, National Institute for Viral Disease Control and Prevention, China CDC, 155 Changbai Road, Beijing, 102206, PR China
}

date, Gluc has been used for the detection and quantification of protein interactions [3], siRNA [4], miRNA [5], promoter activity [6], and viral infection and replication [7]. Furthermore, the Gluc reporter system has also been used in live animal studies [8], and use of a bioluminescence detector [9] facilitated imaging of tumor cell trafficking and proliferation in vivo.

Influenza viruses are members of the family Orthomyxoviridae and are composed of three types: A, B, and C. They possess a single-stranded, negative-sense RNA genome consisting of eight (influenza A \& B) or seven (influenza $C$ ) segments. Formation of a viral polymerase complex consisting of PB2, PB1, and PA proteins together with NP is required for successful replication [10]. Twelve conserved nucleotides at the $3^{\prime}$, and 13 at the 5' UTR regions of vRNA are essential for polymerase recognition and binding for type A virus. Lutz and co-workers [11] introduced influenza A virus-inducible reporter gene

\section{() Biomed Central}


segments (VIRGS, 3' and 5'UTR sequences of the A/WSN/33 NP segment) into a Fluc reporter system to detect and quantify RNP activity. This system has been used to study influenza virus RNP activity by transfection or viral infection of susceptible cells.

Co-transfecting cells with four plasmids encoding PB2, $\mathrm{PB} 1, \mathrm{PA}$, and NP and a reporter gene avoids the generation of infectious virons and a BSL-3 facility for handling the highly pathogenic avian influenza virus. Moreover, RNP activity testing by viral infection of susceptible cells could be mandated when RNP expression plasmids are unavailable. However, a relatively weaker regulation mediated by virus infection directly than that by polymerase complex transfection limited the application of Fluc system. Therefore, it is necessary to develop a rapid, sensitive, quantitative, and safe assay for determining influenza virus polymerase activity. Here, we report a secreted influenza viral UTR-driven Gaussia luciferase (Gluc) reporter system for RNPs function assay.

\section{Materials and methods \\ Plasmids}

Plasmid pAAV-Gluc-Fluc (provided by Dr. Xiaobing $\mathrm{Wu}$ ) was used as the template for Gluc reporter gene amplification. The primers used are listed in Table 1. After purification using the QIAquick Gel Extraction Kit (Qiagen, Valencia, CA, USA), PCR products were cloned into vectors containing the RNA polymerase I promoter and terminator using the In-Fusion(tm) Advantage PCR Cloning Kit (Clontech, Mountain View, CA, USA) according to the manufacturer's instructions. Plasmid of polI-Gluc was sequenced to ensure the absence of unwanted nucleotide changes.

All eight gene segments of influenza viruses A/Quail/ HK/G1/97 (H9N2, G1) and BH Gs/QH/1/2005 (H5N1, $\mathrm{QH})$ were amplified by RT-PCR and cloned into a modified version of the bidirectional expression plasmid pCQI, derived from $\mathrm{pHW} 2 \mathrm{~K}$. A mutation resulting in a change of lysine (K) to glutamic acid (E) was introduced into the PB2 gene of QH strain; this virus was named $\mathrm{QH}$ (PB2-627E). The reporter plasmid pYH-Fluc was kindly provided by Erich Hoffmann.

\section{Cells}

Human embryonic kidney (293T) and human type II alveolar epithelial (A549) cells were maintained in
Dulbecco's modified Eagle's medium (DMEM; Invitrogen, Carlsbad, CA, USA) supplemented with $10 \%$ fetal bovine serum (FBS; Invitrogen), glutamine ( $2 \mathrm{mM}$; Invitrogen), HEPES (10 mM; Invitrogen), penicillin (100 units $/ \mathrm{ml})$, and streptomycin $(100 \mu \mathrm{g} / \mathrm{ml}$; Invitrogen $)$ and incubated in a humidified atmosphere of $5 \% \mathrm{CO}_{2}$ at $37^{\circ} \mathrm{C}$.

\section{Virus preparation and titration}

Recombinant viruses G1, QH (PB2-627K), and QH (PB2-627E) were maintained as reported previously $[12,13]$. Viral titrations were determined using A549 cells, and the tissue culture infectious dose affecting $50 \%$ of the cells $\left(\mathrm{TCID}_{50}\right)$ was calculated using the Reed-Muench formula [14]. All experiments involving live $\mathrm{H} 5 \mathrm{~N} 1$ viruses were performed in a BSL-3 facility.

\section{Transfection and virus infection for RNP activity measurement}

Reporter plasmids pYH-Fluc $(0.1 \mu \mathrm{g})$ and polI-Gluc $(0.1 \mu \mathrm{g})$ were co-transfected with expression plasmids encoding PB2, PB1, PA, and NP into 293T cells $\left(1 \times 10^{5}\right.$ and $5 \times 10^{4} /$ well in 24- and 96-well plates, respectively) using the PolyFect (Qiagen) reagent according to the manufacturer's instructions. Mock transfections were performed with either pYH-Fluc or polI-Gluc alone, as appropriate. For assay RNP activity by virus infection, A549 cells $\left(1 \times 10^{5} /\right.$ well $)$ were transfected with polIGluc, again using the PolyFect reagent. After $6 \mathrm{~h}$ incubation at $37^{\circ} \mathrm{C}$, cells were washed with phosphate buffered saline (PBS), and then the virus was introduced at a multiplicity of infection (m.o.i.) of either 1 or 0.001 . Mock infections were performed using sterile culture medium.

Inhibition of influenza A virus infection by amantadine was assessed by incubating polI-Gluc- or pYHFluc-transfected A549 cells with $0.21 \mu \mathrm{M}$ amantadine for $30 \mathrm{~min}$ prior to the addition of the virus. After incubation for $1 \mathrm{~h}$ at $37^{\circ} \mathrm{C}$, supernatant was decanted, and cells were washed with PBS and then incubated at $37^{\circ} \mathrm{C}$ in DMEM containing amantadine.

\section{Luciferase assay}

Gluc activity in supernatants (cell-free media; $20 \mu \mathrm{l}$ ) was analyzed using a Gluc assay Kit (New England Biolabs, Beverly, MA, USA). Transfected or infected cells were gently washed twice in PBS, followed by the addition of $200 \mu \mathrm{l} 1 \times$ passive lysis buffer (Promega, Madison, WI,

Table 1 Primer sequences used in the construction of the viral UTR-driven Gluc reporter

\begin{tabular}{ll}
\hline Gluc-F & 5'-GGCCGCCGGGTTATTAGTAGAAACAAGGGTATTTTTCTTTAGTCACCACCGGCCCCCTTGATC -3' $^{\prime}$ \\
\hline Gluc-R1 & 5'-CCTCCGAAGTTGGGGAGCAAAAGCAGGGTAGATAATCACTCACAGAGTGACATCGAAAAT-3' \\
\hline Gluc-R2 & 5'-CACAGAGTGACATCGAAAATGGGAGTCAAAGTTCTGTTTGCCCT-3' \\
\hline
\end{tabular}

Bold portions correspond to sequences within the coding region of the reporter gene. The remaining $5^{\prime}$ sequences (15 nt, italics) are necessary for In-Fusion cloning into the RNA polymerase I vector poll-sapl. Underlined parts correspond to the untranslated regions of the A/WSN/33 NP segment. Two reverse primers $(R 1, R 2)$ were designed to ensure PCR efficiency. 
USA). Cell lysates were harvested and centrifuged for $5 \mathrm{~min}$ at 10,000 rpm, and Fluc activity in supernatants (cell lysates; $20 \mu \mathrm{l}$ ) was measured by addition of D-luciferin (100 $\mu \mathrm{l}$; Promega) and by measurement of fluorescence intensity for $10 \mathrm{~s}$ using a Modulus Luminometer machine (Promega).

\section{Statistical analysis}

All determinations were performed in triplicate and repeated three times. Data are expressed as means \pm SEM. Statistical significance was determined using nonparametric tests and the GraphPad Prism 5 software package (GraphPad Software). A $P$-value $<0.05$ was deemed to indicate statistical significance.

\section{Results}

\section{Efficacy of the Gluc reporter system}

A virus-inducible Fluc reporter gene system has been developed and used for quantification of influenza virus polymerase activity [11]. To assess the potential of Gaussia protein as a reporter, we constructed artificial RNA segments encoding the Gaussia protein under the control of the UTRs of influenza A/WSN/33 NP segment. These artificial RNA segments were cloned into the RNA polymerase I promoter/terminator cassette to produce RNA transcripts containing no additional nucleotide sequences or modifications at either the 5' or 3' end. The vector polIGluc and plasmids carrying the PB1, PB2, PA, and NP segments of influenza virus G1 (H9N2) were co-transfected into $293 \mathrm{~T}$ cells, and RNP activity was determined. Around a 600-fold higher luciferase activity was detected in supernatants at $18 \mathrm{~h}$ post-transfection as compared with that in mock-transfected cells (Figure 1A). Furthermore, Gluc signal accumulated with time. In contrast, the Fluc reporter gene signal peaked at $24 \mathrm{~h}$ post-transfection and subsequently remained at this level (Figure 1B).

The efficacy of the Gluc reporter system in assessing viral infection was examined. Little luciferase protein expression was detected from the construct in the absence of an authentic influenza A virus polymerase, similar to luciferase signals of non-transfected cells (data not shown). FluA polI-Gluc-transfected A549 cells were infected with influenza virus G1 (MOI of 1 ), and supernatants were harvested for luciferase analysis at $0,3,6,9,12$, and $24 \mathrm{~h}$ post-infection (p.i.; Figure 1C). Signal was detected as early as 6 h p.i. At 24 h p.i., luciferase activity in G1-infected A549 cell supernatants was 20-fold higher than that in mock-treated cells (Figure 1C). Thus, the Gluc system was capable of quantifying RNP activity and performed similar to the Fluc reporter system.

\section{Sensitivity of Gluc reporter system}

Gluc is $>1000$-fold more sensitive than the American firefly or sea pansy Renilla reniformis luciferases [2]. To

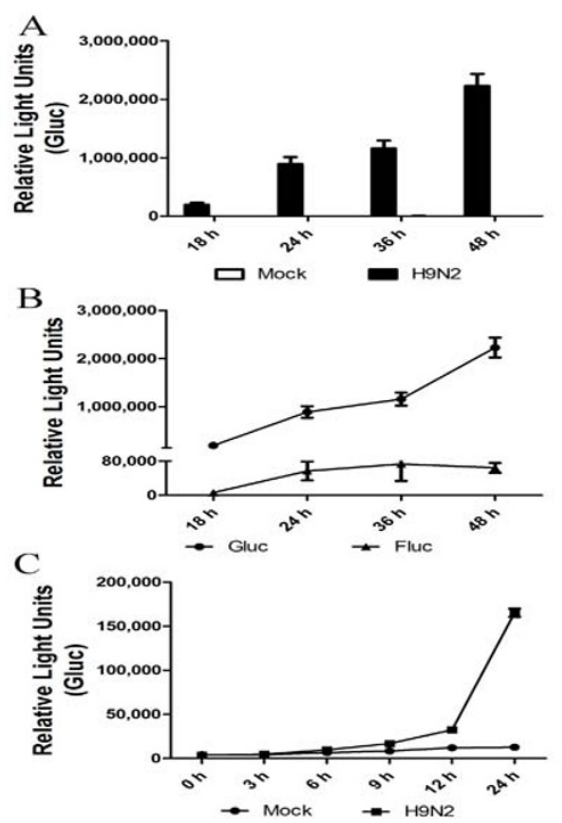

Figure 1 RNP assaying by Fluc and Gluc systems after reconstitution of influenza $A$ virus polymerase complex. (A) Polymerase activity assayed by a viral UTR-driven Gluc reporter gene. (B) Detection and quantification of RNP activity by the Gluc or Fluc reporter systems after transfection. 293T cells were transfected with plasmids encoding the PB2, PB1, PA, and NP genes of A/Quail/HK/G1/97 (H9N2) plus reporter plasmids poll-Gluc or pYH-Fluc. Supernatants (cell-free culture medium) and cell lysates were assayed for luciferase activity using appropriate substrates by a luminometer. (C) Kinetics of G1 RNP activity assayed using the Gluc reporter system after virus infection. A549 cells were transfected with plasmid poll-Gluc and infected at $6 \mathrm{~h}$ post-transfection with influenza virus $\mathrm{G} 1$ at a $\mathrm{MOI}$ of 1. Luciferase activity in supernatants was assayed at 0, 3, 6, 9, 12, and 24 h post-G1 (H9N2) infection and expressed as RLU per well. Data are expressed as standard errors of the mean (SEM) from three independent experiments performed in triplicate.

compare sensitivities of the Gluc versus Fluc reporter systems, we transfected $293 \mathrm{~T}$ cells with six plasmids: the vectors polI-Gluc and pYH-Fluc and plasmids encoding the G1 (H9N2) viral RNP. Supernatants of Gluc-transfected cells showed a 50 -fold higher bioluminescent activity compared with Fluc-transfected cells at $24 \mathrm{~h}$ posttransfection (Figure 1B), again indicating the sensitivity of the Gluc-based system.

\section{Thermostability of Gluc reporter system}

Gluc is stable in culture medium (half-life around $6 \mathrm{~d}$ ) [7], and therefore samples can be stored at $4^{\circ} \mathrm{C}$ for several days without any significant change in Gluc activity. Gluc is also heat stable at $65^{\circ} \mathrm{C}$ [15]. Influenza viruses are not heat-resistant and can be inactivated at $56^{\circ} \mathrm{C}$ for $30 \mathrm{~min}$ or $100^{\circ} \mathrm{C}$ for $1 \min [16,17]$, and at $65^{\circ} \mathrm{C}$ for 30 
min or $100^{\circ} \mathrm{C}$ for 2 min for $\mathrm{H} 5 \mathrm{~N} 1$ virus[18]. We compared Fluc and Gluc activities after heat treatment. Vectors polI-Gluc and pYH-Fluc were introduced together by both transfection and influenza virus infection and supernatants were harvested for Gluc assay and cells lysates for Fluc analysis. All samples were treated at $65^{\circ}$ $\mathrm{C}$ for 10 or $30 \mathrm{~min}$, and then Gluc and Fluc activities were determined. Gluc-transfected supernatants maintained $>90 \%$ activity after treatment at $65^{\circ} \mathrm{C}$ for $10 \mathrm{~min}$ and $>70.0 \%$ after $65^{\circ} \mathrm{C}$ for $30 \mathrm{~min}$. In contrast, Fluc activity decreased to $<10 \%$ after heating for $10 \mathrm{~min}$ and was almost totally absent after 30 min (Figure 2A). Similar results were found by viral infection (Figure 2B).

\section{Determination of optimum infectious dose}

Fluc reporter quantitation was linear in $293 \mathrm{~T}$ cell infected with influenza virus at a MOI of 0.001-0.1 [11]. To elucidate the optimal infectious dose of the Gluc system, we transfected A549 cells $\left(1 \times 10^{5}\right)$ with both pYH-
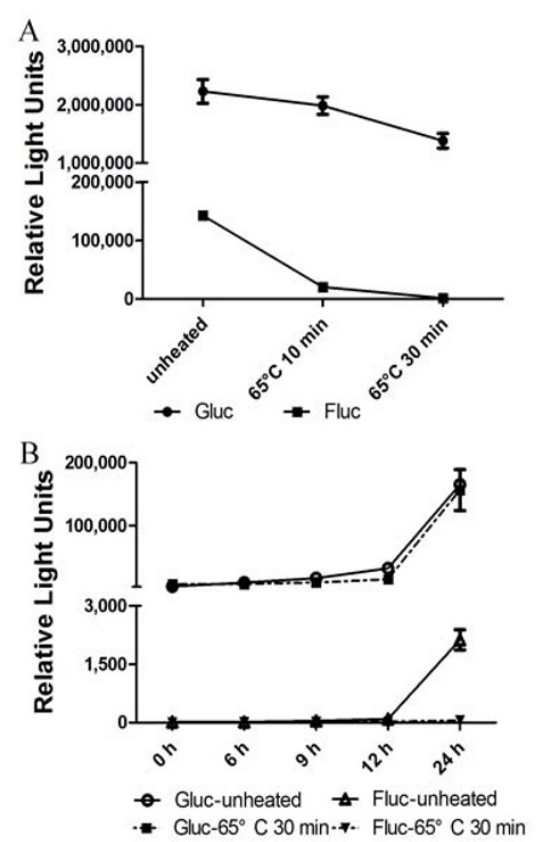

Figure 2 Heat-sensitivity of Gluc and Fluc. (A) RNP activity in poll-Gluc- and pYH-Fluc-transfected 293T cells after heat-treatment. 293T cells were transfected with plasmids encoding the PB2, PB1, PA, and NP segments of influenza G1 (H9N2) plus reporter plasmids poll-Gluc and pYH-Fluc. Supernatants and corresponding cell lysates were heated at $65^{\circ} \mathrm{C}$ for 10 or $30 \mathrm{~min}$, and then Gluc and Fluc activities were assayed. (B) RNP activity in G1-infected A549 cells after heat treatment. Polymerase activity assays were performed in A549 cells transfected with vRNA Gaussia and firefly reporter plasmids and then infected with H9N2 at $6 \mathrm{~h}$ post-transfection. Supernatants and corresponding cell lysates were harvested for assay of Gluc and Fluc activity at appropriate times. Data are expressed as standard errors of the mean (SEM) from three independent experiments performed in triplicate.
Fluc and polI-Gluc, and infected cells with influenza A virus G1 at a MOI of 1 or 0.001 , respectively. Fluc and Gluc activity was assayed following $24 \mathrm{~h}$ incubation at $37^{\circ} \mathrm{C}$ (Figure 3). Gluc (Figure 3B-D), but not Fluc (Figure 3A) activity was significantly different at a MOI of 0.001 as compared with a MOI of 1 . Thus, our findings suggested that a MOI of 0.001 was better than that of 1 for the Gluc reporter assay under these conditions.

\section{Verification of PB2-K627E-induced RNP activity}

A single residue at position 627 in the PB2 subunit of the influenza polymerase (PB2-627) has been reported to be critical for polymerase activity in a species-specific fashion [19-23]. Most human influenza viruses possess a lysine at this position (PB2-627K), and most avian viruses possess glutamic acid (PB2-627E). PB2-627K is correlated with enhanced polymerase activity [19] and the high virulence [24] of H5N1 viruses in mice. PB2$627 \mathrm{E}$ attenuates viral replication efficiency and pathogenicity in mammals [25]. We verified these findings using the Gluc reporter system by both transfection with viral RNPs (Figure 4) and infection with QH (PB2$627 \mathrm{~K}$ ) and QH (PB2-627E) viruses (Figure 3C \& 3D). Signal was stronger in the Gluc than in the Fluc reporter system. A significant difference between PB2-627K and PB2-627E, which was consistent with other reports, was detected using the Gluc reporter system at a MOI of 0.001 , but not at a MOI of 1 (Figure 3C \& 3D). Furthermore, this effect was blocked by addition of amantadine, an influenza virus entry inhibitor (Figure 3B-D; $P<0.05)$.

\section{Discussion}

The influenza virus RNA-dependent RNA polymerase complex subunits (PB1, PB2, and PA) and NP catalyze both genome replication and transcription. This complex is also involved in influenza virus host adaptation [26]. In particular, a mutation in residue 627 of the PB2 segment (glutamic acid to lysine) is necessary for avian and swine influenza viruses to infect humans [27]. The H5N1 reassortant virus that contains a PB2 segment from human seasonal H3N2 is more virulent than the parental H5N1 strain in a mouse model [28]. Recently, a number of laboratories use Fluc reporter system to detect RNP activity. As for the limitations of Fluc system for RNP assay by virus infection, a system for determining influenza virus polymerase activity which would be more sensitive, easier to handle and allow more widespread use is essential.

Here, we first introduced Gaussia luciferase into a viral UTR-driven reporter system. Gaussia luciferase is a monomeric protein from the copepod marine organism Gaussia princeps. The small size of this gene (555 bp), together with its relative lack of toxicity, makes it 

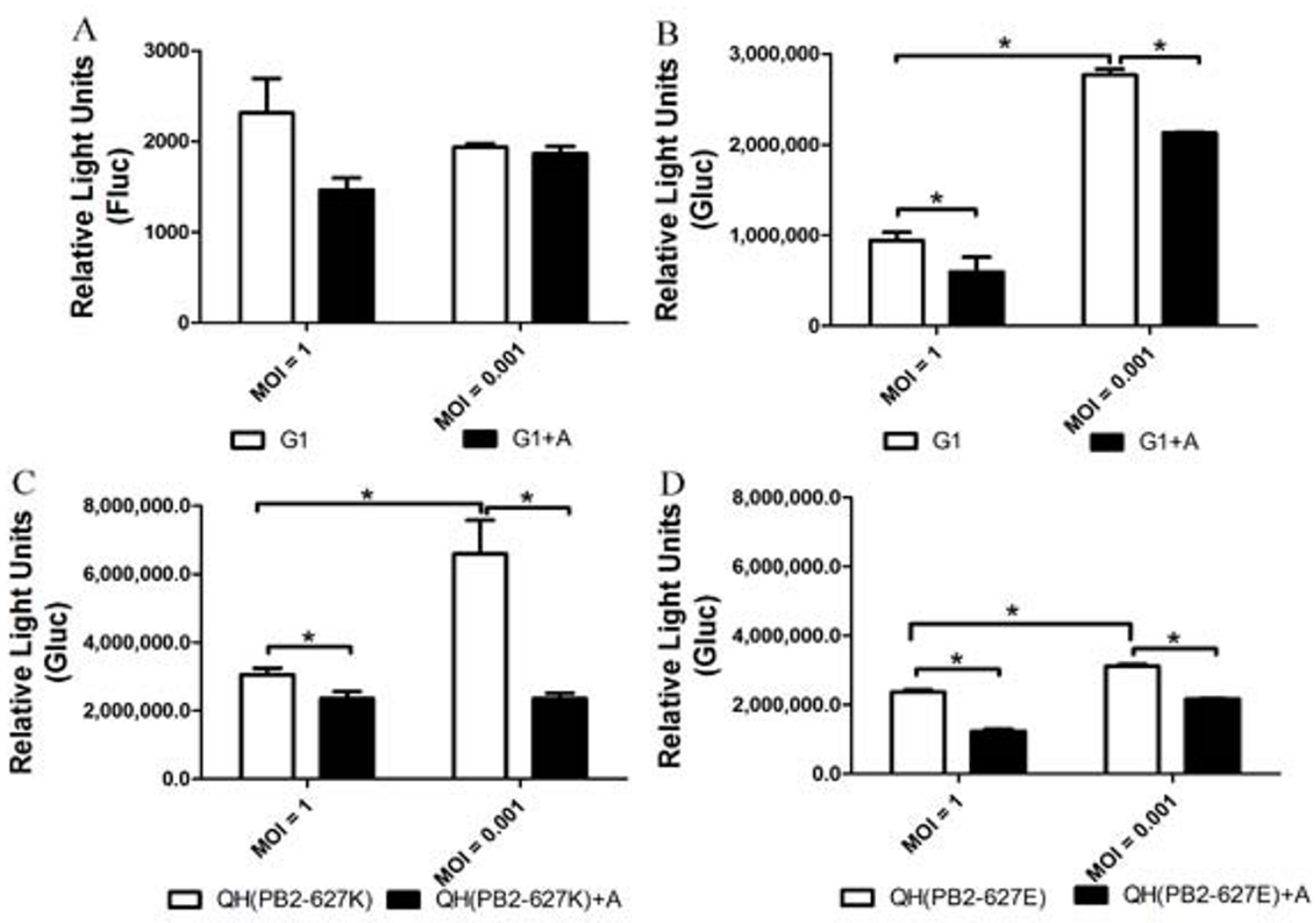

Figure 3 The effect of virus dose and addition of antivirals on RNP activity. A549 cells were transfected with plasmids pYH-Fluc (A) or pollGluc (B, C, and $\mathbf{D})$ and incubated with or without amantadine $(0.21 \mu \mathrm{M})$ for $30 \mathrm{~min}$ at $6 \mathrm{~h}$ post-transfection. Infections were performed with recombinant viruses G1 (A, B), QH (PB2-627K) (C), or QH (PB2-627E) (D) at a MOl of 1 and 0.001. At 24 h p.i., cells were lysed, and Fluc (A) and Gluc activity (B-D) were assayed. Data are expressed as standard errors of the mean (SEM) from three independent experiments performed in triplicate. A, amantadine; ${ }^{*} P<0.05$.

suitable for vector construction and application. Moreover, Gaussia luciferase is naturally secreted and has a broad $\mathrm{pH}$ optimum as compared with other luciferases, with peak activity at pH 7.7 [29]. The Gluc reporter gene was activated and expressed via reconstitution of influenza A virus polymerase complex by either transfection or direct viral infection (Figure 1). The Gluc reporter produced a strong signal that accumulated with time (600- and 20-fold higher than controls in transfected and virus-infected supernatants, respectively; Figure 1), while Fluc signals saturated at $24 \mathrm{~h}$ p.i. with similar observation reported by Sun et al [30]. Furthermore, the Gluc reporter gene system was both much more sensitive and more heat stable than the Fluc system (Figs. 1B \& 2B).

Fluc was activated by G1 (H9N2) infection, but did not differ between one (MOI of 1) and multiple infection cycles (MOI of 0.001). Additionally, no significant effect of amantadine on G1 vRNP activity was found at neither MOI of 1 nor 0.001 (Figure 3A). Notably, stronger signal was detected at MOI of 0.001 than that of 1 for the Gluc reporter assay. Furthermore, signal produced by the Gluc system at MOI of 1 or 0.001 could be modified by addition of amantadine, an influenza virus ion blocker (Figure 3B-D), implying the powerful potency of Gluc system for RNP assay and the screening of antiviral compounds.

Similarly, significant differences between induction by PB2-627K and PB2-627E were detected by both the Gluc and Fluc reporter systems after reconstitution of viral RNPs by transfection (Figure 4), as well as after multiple infection cycles using the Gluc reporter system (Figure 3C-D). Our data suggest that multiple infection cycles might be better for the Gluc reporter system, which may be explained by the efficiency of infection at a low concentration of virus and the resultant amplification of the Gluc signal, showing that the effects of PB2-627K/E or amantadine on influenza vRNP activity were elucidated more effectively by the Gluc reporter system. 


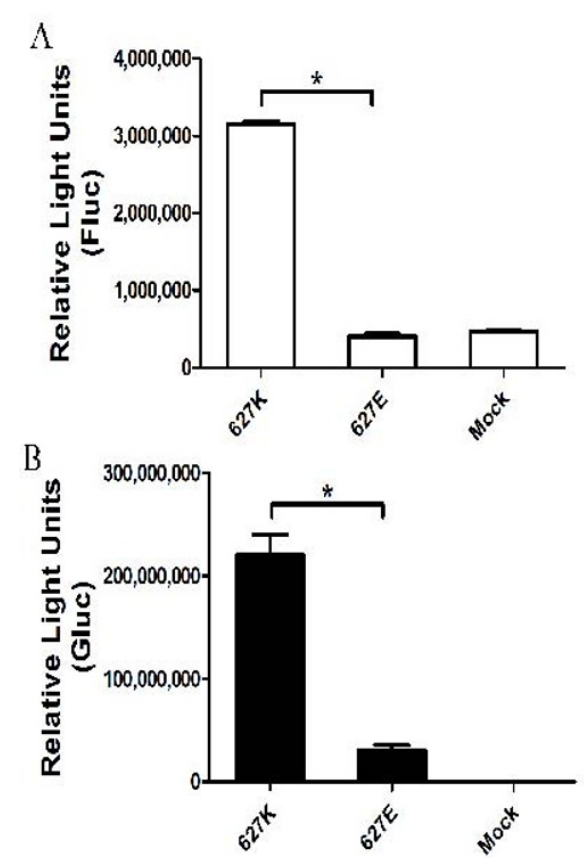

Figure 4 RNP activity of QH virus containing PB2-627K and PB2-627E. Fluc (A) and Gluc (signaling was detected after 1:10 dilution; B) were expressed from a synthetic viral RNA respectively when the influenza virus polymerase complex was present. 293T cells were transfected in 96- (Gluc; $5 \times 10^{4} /$ well) and 24-well (Fluc; 1 $\times 10^{5} /$ well) plates. Control cells were mock transfected with the reporter plasmid alone. Data are expressed as standard errors of the mean (SEM) from three independent experiments performed in triplicate. ${ }^{*} P<0.05$.

\section{Conclusion}

Generally, Gluc system constructed here provided an alternative method for evaluating influenza virus polymerase activities, and showed much more advantages than traditional Fluc system. Firstly, Gluc luciferase was naturally secreted from cells into culture medium and could be detected readily. Secondly, much stronger signal intensity was generated by Gluc system. Finally, besides of its heat-resistance, Gluc reporter system works better during viral infection, which is suitable for RNP assay by direct virus infection and avoids constructing RNP plasmids. The reporter system based on secreted Gaussia luciferase activity for assaying influenza virus RNP functionality is sensitive, easier-detectable, and thermo-stable and is a potential tool for influenza virology study, especially for highly pathogenic avian influenza virus.

\section{Abbreviations}

Gluc: Gaussia luciferase; Fluc: firefly luciferase; BSL-3: biosafety level 3 laboratory; vRNP: viral ribonucleoprotein; PB2: polymerase basic 2 protein; PB1: polymerase basic 1 protein; PA: polymerase acidic protein; NP: nucleoprotein; PCR: polymerase chain reaction; UTR: untranslated region; p.i.: post-infection; MOI: multiplicity of infection; VIRGS: virus-inducible reporter genes

\section{Acknowledgements}

We thank Erich Hoffmann for kindly providing the reporter plasmid $\mathrm{pYH}$ Fluc

This study was supported by the National Basic Research (973)

(2011CB504704), the National Special Program for Infectious Disease Control and Prevention (2008ZX10004-013), and the National Science and

Technology Major Project "Development of Novel Vaccines, Antibodies and Therapeutic Agents against Influenza Infection" (2009ZX10004-016).

\section{Authors' contributions}

SYL and ZJF designed research. ZWF performed research. SYL contributed new reagents/analytic tools. ZWF and ZJF analyzed data and wrote the paper. QK, YZJ, ZY and TWH helped to construct partial plasmids. QK and DN helped to draft the manuscript. LLQ and WXB provided partial plasmids. All authors read and approved the final manuscript.

\section{Competing interests}

The authors declare that they have no competing interests.

Received: 28 October 2010 Accepted: 21 January 2011

Published: 21 January 2011

\section{References}

1. de Wet JR, Wood KV, Helinski DR, DeLuca M: Cloning of firefly luciferase CDNA and the expression of active luciferase in Escherichia coli. Proc Natl Acad Sci 1985, 82:7870-7873.

2. Tannous BA, Kim DE, Fernandez JL, Weissleder R, Breakefield XO: Codonoptimized Gaussia luciferase cDNA for mammalian gene expression in culture and in vivo. Mol Ther 2005, 11:435-443.

3. Remy I, Michnick SW: A highly sensitive protein-protein interaction assay based on Gaussia luciferase. Nat Methods 2006, 3:977-979.

4. Morlighem JE, Petit C, Tzertzinis G: Determination of silencing potency of synthetic and RNasell generated siRNA using a secreted luciferase assay. Biotechniques 2007, 42:599-600.

5. Lee JY: Development of a dual-luciferase reporter system for in vivo visualization of microRNA biogenesis and posttranscriptional regulation. J NuCl Med 2008, 49:285-294.

6. Ruecker O, Zillner K, Groebner-Ferreira R, Heitzer M: Gaussia-luciferase as a sensitive reporter gene for monitoring promoter activity in the nucleus of the green alga Chlamydomonas reinhardtii. Mol Genet Genomics 2008, 280:153-162.

7. Wurdinger T, Basr C, Pike L, de Kleine R, Weissleder R, Breakefield XO, Tannous BA: A secreted luciferase for ex vivo monitoring of in vivo processes. Nat Methods 2008, 5:171-173.

8. Asokan A, Johnson JS, Li C, Samulski RJ: Bioluminescent virion shells: New tools for quantitation of AAV vector dynamics in cells and live animals. Gene Ther 2008, 15:1618-1622.

9. Tannous BA: Gaussia luciferase reporter assay for monitoring of biological processes in culture and in vivo. Nat Protoc 2009, 4:582-591.

10. Lamb RA, Krug RM: Orthomyxoviridae: The viruses and their replication. Fields Virology 2007, 1647-1689.

11. Lutz A, Dyall J, Olivo PD, Pekosz A: Virus-inducible reporter genes as a tool for detecting and quantifying influenza $A$ virus replication. J Virol Methods 2005, 126:13-20.

12. Fodor E, Devenish L, Engelhardt OG, Palese P, Brownlee GG, García-Sastre A: Rescue of influenza A virus from recombinant DNA. J Virol 1999, 73:9679-9682.

13. Steel J, Burmakina SV, Thomas C, Spackman E, García-Sastre A, Swayne DE, Palese P: A combination in-ovo vaccine for avian influenza virus and Newcastle disease virus. Vaccine 2008, 26:522-531.

14. Reed $L$, Muench $\mathrm{H}$ : A simple method of estimating fifty percent endpoints. Am J Hyg 1938, 27:493-497.

15. Cullen BR, Malim MH: Secreted placental alkaline phosphatase as a eukaryotic reporter gene. Methods Enzymol 1992, 216:362-368.

16. Shahid MA, Abubakar M, Hameed S, Hassan S: Avian influenza virus (H5N1); effects of physico-chemical factors on its survival. Virol J 2009, 6:38.

17. Influenza C, influenza C virus. [http://influenzac.com]. 
18. Avian influenza. [http://baike.baidu.com/view/266587.html].

19. Subbarao EK, London W, Murphy BR: A single amino acid in the PB2 gene of influenza A virus is a determinant of host range. J Virol 1993, 67:1761-1764.

20. Le QM, Ito M, Muramoto Y, Hoang PV, Vuong CD, Sakai-Tagawa Y, Kiso M, Ozawa M, Takano R, Kawaoka Y: Pathogenicity of highly pathogenic avian H5N1 influenza A viruses isolated from humans between 2003 and 2008 in northern Vietnam. J Gen Virol 2010, 91:2485-2490.

21. Li YG, Chittaganpitch M, Waicharoen S, Kanai Y, Bai GR, Kameoka M, Takeda N, Ikuta K, Sawanpanyalert P: Characterization of H5N1 influenza viruses isolated from humans in vitro. Virol I 2010, 1:112.

22. Shinya K, Makino A, Ozawa M, Kim JH, Sakai-Tagawa Y, Ito M, Le QM, Kawaoka Y: Ostrich involvement in the selection of $\mathrm{H} 5 \mathrm{~N} 1$ influenza virus possessing mammalian-type amino acids in the PB2 protein. J Virol 2009, 83:13015-13018.

23. Fornek JL, Gillim-Ross L, Santos C, Carter V, Ward JM, Cheng LI, Proll S, Katze $M G$, Subbarao $K$ : A single-amino-acid substitution in a polymerase protein of an $\mathrm{H} 5 \mathrm{~N} 1$ influenza virus is associated with systemic infection and impaired T-cell activation in mice. J Virol 2009, 83:11102-11115.

24. Hatta M, Gao P, Halfmann P, Kawaoka Y: Molecular basis for high virulence of Hong Kong H5N1 influenza A viruses. Science 2001, 293:1840-1842.

25. Shinya $K$, Hamm S, Hatta M, Ito $H$, Ito $T$, Kawaoka Y: PB2 amino acid at position 627 affects replicative efficiency, but not cell tropism, of Hong Kong H5N1 influenza A viruses in mice. Virology 2004, 320:258-266.

26. Yamada S, Hatta M, Staker BL, Watanabe S, Imai M, Shinya K, SakaiTagawa Y, Ito M, Ozawa M, Watanabe T, Sakabe S, Li C, Kim JH, Myler PJ, Phan I, Raymond A, Smith E, Stacy R, Nidom CA, Lank SM, Wiseman RW, Bimber BN, O'Connor DH, Neumann G, Stewart LJ, Kawaoka Y: Biological and structural characterization of a host-adapting amino acid in influenza virus. PLOS Pathog 2010, 6.

27. Mehle A, Doudna JA: Adaptive strategies of the influenza virus polymerase for replication in humans. Proc Natl Acad Sci 2009, 106:21312-21316.

28. Li C, Hatta M, Nidom CA, Muramoto Y, Watanabe S, Neumann G, Kawaoka Y: Reassortment between avian H5N1 and human H3N2 influenza viruses creates hybrid viruses with substantial virulence. Proc Natl Acad Sci 2010, 107:4687-4692.

29. Szent-Gyorgyi C, Ballou BT, Dagmal E, Bryan B: Cloning and characterization of new bioluminescent proteins. Proceedings of SPIE 1999, 3600:4-11.

30. Sun ZD, Sun P, Fu QX, Wang YZ, Zhou Y, Peng JC, Zhan LS: The agreement study of the different methods used for detecting the firefly luciferase activity. Letters in biotechnology 2008, 19:80-83.

doi:10.1186/1743-422X-8-29

Cite this article as: Zhu et al:: A reporter system for assaying influenza virus RNP functionality based on secreted Gaussia luciferase activity.

Virology Journal 2011 8:29.

\section{Submit your next manuscript to BioMed Central and take full advantage of:}

- Convenient online submission

- Thorough peer review

- No space constraints or color figure charges

- Immediate publication on acceptance

- Inclusion in PubMed, CAS, Scopus and Google Scholar

- Research which is freely available for redistribution

Submit your manuscript at www.biomedcentral.com/submit
CioMed Central 